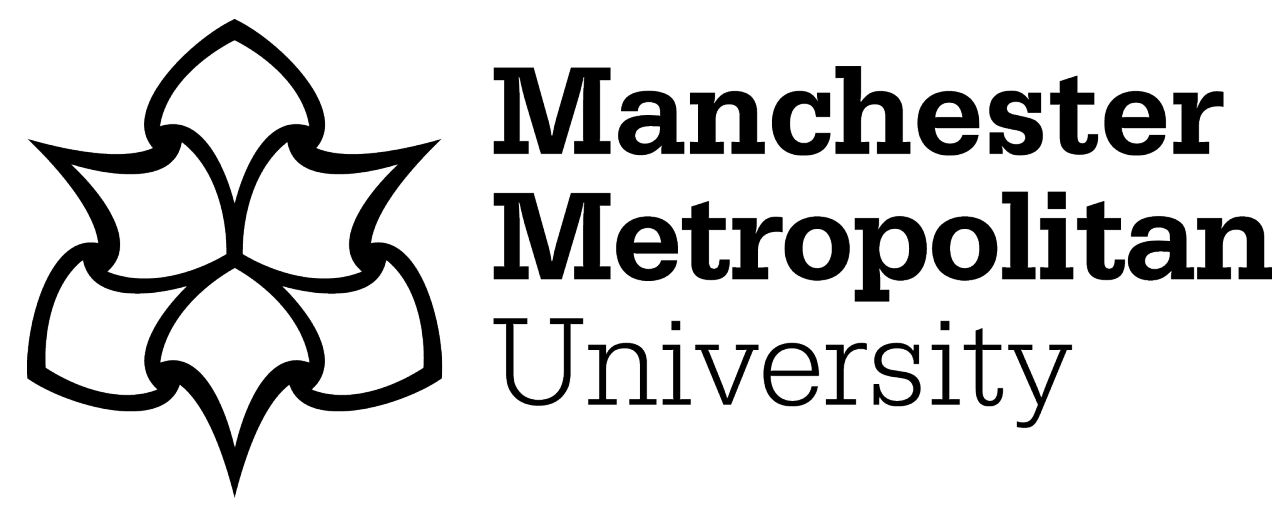

Djahel, Soufiene ORCID logoORCID: https://orcid.org/0000-0002-12867037, Jabeur, Nafaa, Nait-Abdesselam, Farid and Wolstencroft, Thomas (2021) A WAVE based and Collaboration driven Framework for Reduced Traffic Congestion in Smart Cities. IEEE Intelligent Transportation Systems Magazine, 13 (4). pp. 251-261. ISSN 1939-1390

Downloaded from: https://e-space.mmu.ac.uk/623877/

Version: Accepted Version

Publisher: Institute of Electrical and Electronics Engineers

DOI: https://doi.org/10.1109/MITS.2019.2953487

Please cite the published version 


\title{
A WAVE based and Collaboration driven Framework for Reduced Traffic Congestion in Smart Cities
}

\author{
Soufiene Djahel ${ }^{\mp}$, Nafaa Jabeur ${ }^{ \pm}$, Farid Nait-Abdesselam ${ }^{\$}$ and Thomas Wolstencroft ${ }^{\mp}$ \\ ${ }^{\mp}$ Department of Computing and Mathematics, Manchester Metropolitan University, UK \\ ${ }^{ \pm}$German University of Technology in Oman, Sultanate of Oman \\ ${ }^{\$}$ CSEE Department, University of Missouri Kansas City, USA
}

\begin{abstract}
The fast emergence of autonomous vehicles promises a drastic change on how road traffic congestion is detected, controlled, and mitigated. To this end, we believe that it is beneficial to explore different ways of collaboration between autonomous vehicles, with the aid of modern road infrastructure, to optimize the commuters' travel time. We, therefore, propose in this paper a novel solution based on a multi-parties collaboration framework and built upon the WAVE standard to optimize the usage of the road network and lower commuters' travel time. Our solution, which is based on a Belief-Desire-Intention architecture, enables autonomous vehicles to opt for selfish or collaborative behaviors depending on their goals and current situations. The results obtained from our preliminary prototype under three representative road maps demonstrate the effectiveness of our approach in dealing with traffic congestion.

Keywords - Smart transportation, road traffic congestion, vehicular communication, multi-parties collaboration.
\end{abstract}

\section{INTRODUCTION}

The concept of Smart City continues to attract high attentions worldwide. Its successful implementations in many cities, including Amsterdam, Barcelona, Milton Keynes, and Stockholm is motivating governmental, industrial, academic and social actors to join their efforts and build sustainable ecosystems promoting citizen welfare and economic growth. Smart City is mainly based on advanced ubiquitous ICT technologies to monitor the city assets, such as energy, water, and transportation infrastructure. In a smart city context, advanced and efficient technology-based monitoring of critical infrastructures is predominant to make the city more sustainable while promoting its economic development. Smart transportation is considered a key enabler of such growth since the efficiency of several other services are reliant on its robustness. Such robustness cannot be achieved without developing advanced solutions to deal with traffic congestion problems and their resulting impact on the commuters' travel experience and safety.

In the context of transportation management, the problem of traffic congestion is being addressed by enabling appropriate V2X (Vehicle-to-everything) communications based on realtime data collected by smart sensors. These sensors are commonly deployed on vehicles or in well-selected locations along roads. Although important progress is being made, traffic congestion remains an enduring problem, particularly because current transportation infrastructures are not being expanded as fast as the growing human and goods transportation needs in modern cities. A study conducted by Texas Transportation Institute has shown that, in 2014, the economic loss due to road traffic congestion in terms of additional travel delay and fuel consumption was almost $\$ 160$ billion [1]. During this period, American drivers traveled an extra 6.9 billion hours and purchased an additional 3.1 billion gallons of fuel as a consequence of traffic congestion.

Congestion normally occurs when a section of a road is overflowing with traffic, resulting thereby into extended trip durations, slower speeds, and longer cars queuing. Congestion can also occur during ongoing road maintenance, making it difficult for vehicles to navigate the road normally. The two congestion cases above are known as recurring and nonrecurring. Non-Recurring congestions are particularly difficult to cope with because they occur when sporadic events (e.g., road accidents, environmental accidents, festivals and road maintenance) take place.

Due to the fast growing urbanization worldwide causing the number and complexity of recurring and non-recurring congestions to considerably increase, more appropriate congestion management systems, responding to the specific needs of smart cities, are needed. To this end, we propose in this paper an advanced system where intelligent mechanisms are embedded on-board of vehicles to boost their decision-making process and optimize their collaboration toward a better controlling of road traffic congestion and an improved commuters' travel experience. Our contributions could be summarized as follows: (1) developing a novel collaboration mechanism between smart cars and advanced road infrastructure; (2) supporting this mechanism by proposing an extended Belief-Desire-Intention framework; and (3) adaptive driving policies depending on traffic conditions.

The remainder of the paper is organized as follows. Section II reviews the existing works that have addressed the management of road traffic congestion. Section III presents the fundamentals of our solution along with its detailed operation. In Section IV, we evaluate the performance of our solution and analyze the obtained simulation results. Finally, we conclude in Section V. 


\section{RELATED WORK}

Reducing recurrent road traffic congestion and mitigating their overwhelming consequences are still challenging problems for traffic authorities as well as the transportation research community. For instance, intensive efforts are being made to eliminate the congestion causes, mitigate congestion effects, maintain safe driving, and lower environmental impact. The solutions presented in [2] cover the different phases of modern Traffic Management Systems (TMSs), ranging from advanced data collection techniques and technologies, route planning and re-routing solutions, and short to medium term traffic forecast approaches to smart parking reservation and management systems. Among the most recent significant contributions, Wang et al. [3] have designed a multi-agent based solution called Next Road Rerouting (NRR) that provides drivers with the most suitable next road choice, thereby minimizing the incurred delay after the occurrence of an enroute event such as car crashes and other unpredictable events. To further improve the efficiency of NRR, the authors have proposed the Adaptive NRR (A-NRR) [4] approach in which an automatic and smart calibration of NRR's algorithmic and operational parameters is achieved using a coefficient of variation based method and K-means algorithm. Moreover, the inputs of induction loops were replaced by traffic information acquired through Vehicular Ad-hoc Networks (VANETs) capabilities (since VANETs can cover larger road network area and provide richer traffic information at higher update rate). To ensure that future traffic congestion solutions developed for autonomous vehicles are more accurately evaluated, a new reinforcement learning based computational framework was proposed in [5]. Its major aim is to support the rapid growth of autonomy in ground traffic and enable the development of reliable controllers for complex problems, such as controlling mixed-autonomy traffic in a ring road.

In addition to the above-mentioned works, many simple and/or practical solutions have been proposed and implemented to reduce traffic congestion in and around several cities. In this perspective, parking cash-outs ${ }^{1}$ and free parking at work (e.g., [6]) have been proposed as parts of solutions to traffic congestion wherein financial incentives are offered by companies to their employees to leave their vehicles at home and use alternative public transport means. Patrick Siegman 2 has presented a study where data is collected from 10 different parking cash-out cases covering 50,000 employees. The analysis of this data revealed that an average payment of $\$ 46$ per month to each employee yields an average decrease of $25 \%$ in parking demands. The proposed study has also suggested that traffic congestion could be lowered if cashout solutions are implemented appropriately. Mobile Transit Society (MTC) has proposed to mitigate the impact of traffic congestion by abolishing automobile subsidies (such as free parking) in towns and cities in order to encourage the usage of alternative and environment friendly transportation means. MTC has also proposed to introduce the so-called Fare Lanes wherein instead of imposing a tax to use a road (i.e. tolls) a

\footnotetext{
${ }^{1}$ http://www.bestworkplaces.org/pdf/ParkingCashout_07.pdf

${ }^{2} \mathrm{http}: / /$ moderntransit.org/cashout/cashout.html
}

vehicle user installs a "Tag" device that transmits to a roadside "reader" the vehicle details whenever a fare lane is used. A monthly bill is then issued and sent to the driver for the total cost for using the fare lanes. Here, the readers may apply different fares based on the vehicle category (e.g. buses, trucks, taxi, regular vehicle, etc.). Ultimately, the proposed solution aims to create less congested lanes offering lower travel time at higher costs.

Despite the variety of techniques being used to deal with road traffic congestion, little attention only has been paid to the potential of boosting the collaboration between smart cars and road infrastructure to achieve better control of traffic congestion. This shortcoming is the motivation behind our current work where we present a solution that leverages such collaboration and ultimately improve commuters' travel experience in congested roads. Moreover, our solution has the potential to improve and extend some existing applications provided by car manufacturers as discussed below.

The German automaker BMW was the first to launch an application called EnLighten that displays to the driver the status of the traffic light in front of the car and predicts when this signal will change. In this case, drivers can start braking earlier to prevent sharp braking when traffic lights suddenly change. This application will certainly make driving safer and economic by reducing the number of unnecessary acceleration/deceleration. This application uses iPhone's GPS data as well as Traffic Management Systems data in some cities to ensure accurate prediction of the remaining time for the traffic light signal to change. Our proposed solution (or at least one of its main features) can be easily integrated with this application, if adequate smart traffic lights are in place, to make the journey of future smart cars (or self-driving cars) safer, faster, and more economic. Indeed, this aim is highly achievable at mid-term as the age of self-driving cars is approaching very fast and gaining an increasing acceptance by the drivers' community, especially in emerging markets like China and India [7].

\section{Proposed Solution}

In this section, we will present the key idea of our solution, its operational details as well as the proposed multi-parties collaboration framework.

\section{A. Fundamentals of the proposed solution}

Basically, our main idea is about finding an efficient way of collaboration between smart cars and advanced road infrastructure (such as advanced Traffic Light Controllers (TLCs)). More specifically, the ultimate goal of our idea is to guarantee better mitigation and monitoring of road traffic congestions resulting from random en-route events (i.e. non-recurrent congestion) or the excessive number of cars in road networks (i.e. recurrent congestion). The idea is supported by a cognitive radiolike approach. The approach is inspired from cognitive radio principle used in wireless networks [8]. According to this principle, wireless devices, thanks to their smart transceivers, are able to detect and opportunistically use the unused frequency bands in their vicinity. Similarly, our approach enables 
smart cars to opportunistically use underutilized reserved lanes on the roads, based on certain policies and under certain circumstances. In our design, the role of smart transceivers is ensured by the TLCs that monitor the road traffic, detect the underutilized reserved lanes (e.g., bus lanes) and regular lanes, and take/recommend actions to best balance the road traffic and mitigate possible traffic jams and bottlenecks.

Our approach, which is depicted in Figure 2 (see Section III.C for more details), can be implemented by embedding intelligent mechanisms into communicating devices, including cars and road traffic monitoring equipment. Thanks to the recent advances in vehicular communications, the use of multiradio Wireless Access in Vehicular Environments (WAVE) devices over multi channels (i.e. Control Channel $(\mathrm{CCH})$ and the set of available Service Channels (SCHs)) will lead to a drastic shift of inter-vehicle communications' reliability [9]. Consequently, more efficient coordination between neighboring vehicles could be achieved. This coordination is particularly important as it allows vehicles to take the right actions at the right moments (such as accelerating/decelerating, changing lane or direction) to serve their individual needs as well as the needs of neighboring vehicles, while avoiding any breach of the driving safety requirements (e.g. safety distance, speed limits etc.).

In order to implement an efficient coordination, the contextawareness of vehicles should be extended. To meet this goal, we propose to use information received from two types of beacons: Regular beacons and TLC beacons. Regular beacons are broadcast by each vehicle to share its own information, objectives, and actions with other vehicles within its transmission range. They are generated periodically at an adequate transmit rate and power. This rate is continuously adjusted accordingly to the observed channel collision rate and vehicles density in order to ensure higher transmission reliability. TLC beacons are broadcast periodically by each TLC to advertize the remaining time for the current traffic cycle, any update in driving policies as well as any recommendation to vehicles. Each TLC beacon concerns a specific road section. It is also transmitted during regular intervals as follows:

$$
T L C-B_{I}=\frac{\frac{R-s_{l} g_{l}}{\text { max }- \text { speed }}}{R}
$$

Where $T L C-B_{I}$ is the TLC beacon transmission interval, $R-\mathrm{seg}_{l}$ refers to road segment length and max - speed denotes the maximum speed allowed on this road segment. $R$ (i.e. rate) is an integer value representing the minimum number of times the TLC beacon is broadcast while a car is moving at the maximum speed along the road segment. The TLC beacon transmission interval is thoroughly selected based on the contextual information of the road segment (such as length and average speed of vehicles) controlled by the TLC. This ensures that each vehicle will receive a TLC beacon at least $\mathrm{R}$ times before it reaches the next road intersection (next TLC).

In our solution, we assume that the cars (i.e. the drivers) tend to be collaborative in their actions. Each car (ultimately the driver) should, indeed, adjust its current speed (i.e. ac- celerate or decelerate) and move to a new lane, whenever possible, to accommodate as many requests from neighboring cars as possible, as long as its own objectives are met. To this end, vehicles' actions will be decided individually and collaboratively according to our multi-parties collaboration framework presented in Figure 1. In order to boost vehicles' collaboration, individual vehicles could identify selfishly acting neighboring vehicles and exchange information about them by piggybacking their identity into the regular beacon messages, subject to the presence of a sophisticated reputation and trust management system such as REPLACE proposed in [10].

\section{B. Model of Collaboration}

The rapid changes in technology as well as resource scarcity are amplifying our needs to live in an increasingly interconnected world [11]. To accomplish the requirements of this world, collaboration mechanisms and facilities have attracted intensive focus from research and development communities (e.g., [12] and [13]). Several conceptualizations of collaboration have been proposed without agreeing on a common definition. We retain, however, the vision that collaboration is a process involving shared rules and mutually beneficial interactions, in which autonomous or semi-autonomous actors interact through formal and informal negotiation, jointly creating rules and structures governing their relationships and ways to act or decide on the issues that brought them together [14]. Based on this vision, we propose a new model that we illustrate in Figure 1. In what follows, we outline this model and explain its relevance in reducing travel time in a smart city context.

1) Multi-parties Collaboration Framework: In order to allow the vehicles to reason individually about occurring events in their surrounding environments, we proposed in a related work [2] a Belief-Desire-Intention (BDI) architecture, where beliefs represent the local information that a vehicle has about itself (e.g., its current speed, position, and remaining distance to the next intersection) and its environment (including the road infrastructure, neighboring vehicles, and events of interest like announced obstacles/crashes ahead), desires reflect the objectives or the situations that the vehicle would like to accomplish, and intentions refer to the actions that the vehicle has chosen to do. In this paper, we extend and reformulate this architecture by integrating some mechanisms to support collaboration between vehicles.

The initial BDI architecture was focusing on the individual interests of each vehicle and there was no collaboration mechanisms implemented between the different vehicles. The operations of the system were, therefore, not optimized and did not allow any mutual support on tasks which are beyond the individual capabilities of vehicles. In the new architecture, we are endowing each vehicle with mechanisms to decide on whether to act for its own benefits or for the benefits of the group to which it belongs (i.e., the group of vehicles with which the vehicle has some relations such as members of the same cluster). For instance, the vehicle can decide to act selfishly (i.e., according to the setup of the initial architecture) or can aim to achieve the mutual goals and benefits of the 
vehicles with which it is collaborating. To this end, the vehicle exchanges with peers several information, including contextual information, ongoing actions, and plans and then sets up its plan accordingly. To meet this end, we added the component "Administration Management" where the vehicle can assess its costs and benefits, do the necessarily data analytics, and checks its current rules and commitments with peers.

Let us suppose that our system is composed of several distributed, autonomous entities (i.e. vehicles). These entities hold knowledge about themselves as well as about other entities (e.g., the road traffic system and neighboring vehicles) in their surrounding environments. They would need to collaborate in order to cope with any event of interest in the vicinity (e.g., vehicle crash, water accumulated because of rain, etc.). To this end, every vehicle will be always listening to beacons and communications from neighboring vehicles and TLCs. When new information are received, they will be analyzed and the different modules described in our framework (see Figure 1) will be activated accordingly.

If a vehicle is going to plan an individual action (e.g., accelerate, decelerate, change lane, etc.), it generates its own desires (objectives) as well as its own intentions (actions). If it is going to coordinate with third parties, then mutual desires and mutual intentions are generated. In this case, the Plan Generation Module has to combine the individual and mutual intentions in order to generate a plan that will achieve joint objectives (e.g., improve the traffic flow throughput at intersections) while fulfilling the individual goals of the vehicle. After the execution of this plan, the beliefs of the vehicle are revised, the trust on third peers is assessed by the Norms Management Module and new collaboration rules may be created by the Governance Module.

According to our framework (Figure 1), each entity (i.e. vehicle) contains three components: Administration Management (AM), Concurrency Management (CM), and Execution Management (EM). When data is received from a third party (e.g., the TLC or a neighboring vehicle), the AM module will filter the data and assess the costs and the benefits of its individual and collaborative actions. To this end, it analyzes the outcomes of its previous actions and consults its current governance norms to delimit the legitimacy and the scope of its expected actions. The CM module will then concurrently implement a BDI architecture for individuality management (i.e., serving individual benefits) and a BDI architecture for mutuality management (i.e., serving collective benefits). The individuality management module will make the necessary actions to revise the current beliefs, generate the individual options (e.g., accelerate, decelerate, change lane, etc.), and identify the actions (e.g., accelerate) serving the sole benefits of the vehicle. Furthermore, based on the analysis of the AM module, if collaboration is needed, the mutuality management module will revise the shared beliefs, generate shared options to meet shared objectives (e.g., improve the traffic flow throughput at intersections), and determine the actions that will serve the benefits of the collaborating vehicles. Once individual and mutual action plans are made, they are checked for conflicts and a final action plan is generated. This plan is then going to be sent to the EM module. Once the execution finishes, individual and mutual beliefs are revised. Some governance norms could also be revised based on the experience that the vehicle just had with other vehicles.

\section{Operational details: the different steps}

The operation of our solution follows the steps described in Figure 2 in which we distinguish three key components of our design: the master TLC, the smart adaptation engine, and the cluster of vehicles (i.e., the vehicles currently moving on the same road segment). The master TLC is controlling a set of incoming and ongoing lanes. It is responsible for evaluating the traffic conditions in these lanes in terms of Road occupancy Level (RoL) and Average Speed (AvS). It also periodically exchanges its acquired knowledge (contextual data) about local traffic conditions (i.e. traffic conditions on the lanes that the master TLC controls) with its neighboring TLCs (slaves). Upon reception of the external contextual data from its TLC slaves, the master TLC aggregates the received information alongside its local knowledge (collected through induction loops as well as other inputs such as Bluetooth sensors deployed at the corresponding road intersection) to build more accurate information about the traffic conditions on the lanes it controls as well as their surroundings. This aggregation process is necessary to ensure higher efficiency for the measures to be recommended by the Smart Adaptation Engine (see Figure 2).

The Smart Adaptation Engine represents the main component of our solution, as its output will determine the performance of the whole system. It is composed of two subcomponents: Learning Sub-Component (LSC) and Adaptation Sub-Component (ASC). The role of the LSC is to build and continuously update a knowledge base using the traffic conditions information collected by the master TLC. It is also responsible of defining a profile of a set of traffic conditions, including normal conditions. The definition of these profiles takes into account the perception/evaluation of the slave TLCs as well. This knowledge and profiles built and defined by the LSC are then used as input for the ASC to recommend the most appropriate adaptations to apply.

The output of the Smart Adaptation Engine could be one of the following decisions:

- In roads with more than one lane in both driving directions, if high congestion level is observed in one direction whereas the other direction is having a normal traffic load, a decision can be made (when applicable) to increase the number of lanes dedicated to the congested direction, subject to tolerated impact on the traffic conditions on the opposite direction.

- If an occurring en-route event blocks the road, the Smart Adaptation Engine applies a re-routing mechanism to find adequate alternative routes. To this end, similar solutions like in [3] and [4] could be used.

- Temporarily enabling a special driving policy by allowing regular cars to use additional, non-emergency lanes (e.g. lanes initially reserved for buses or carpooling) if they are underutilized or clear (completely empty). To this end, we are proposing cognitive radio-like policy whereby the 


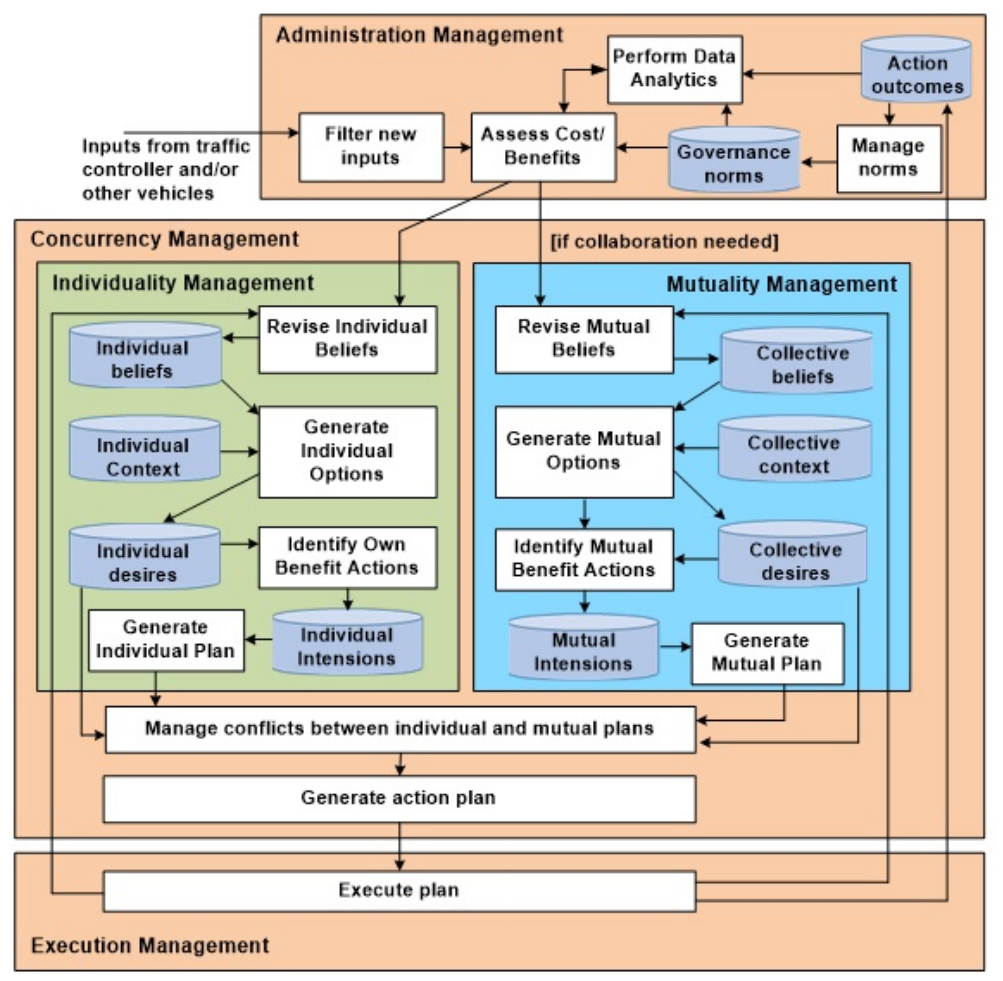

Figure 1: Extended BDI architecture supporting individual and collaborative actions

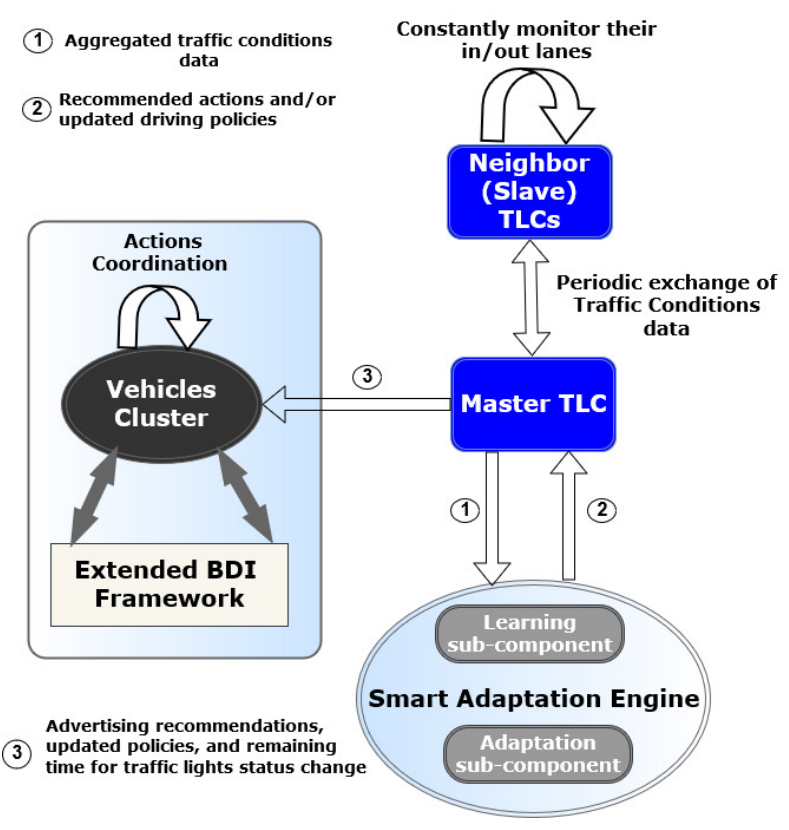

Figure 2: Architecture of the proposed solution

selection of vehicles that will be temporarily permitted to use a reserved lane is made based on car profiles (e.g., eco-friendly, carbon emission, etc.) as well as drivers' profiles (e.g., police, doctors, professors during school time, etc.). In certain circumstances, the driver/car can also send to the TLC an access request to reserved lanes that will be either accommodated or denied based on the usage level of these lanes.
After receiving a TLC beacon carrying the decisions (recommendations) of the Smart Adaptation Engine as well as the remaining time for the current traffic cycle, the vehicles perform actions accordingly while taking into consideration their individual contextual information as well as their individual objectives. Each action is performed in accordance to our extended BDI framework (see Figure 1) described in Section III.B.

\section{Multi-radio based beacons transmission}

In order to allow reliable coordination between vehicles while ensuring safe driving actions, we assume that each vehicle is equipped with two radio transceivers; one is tuned for transmitting periodic beacons and emergency messages on the Control CHannel $(\mathrm{CCH})$ and the other is tuned for sending other types of IP messages on the available Service CHannel(s) $(\mathrm{SCH})$. In this way, the $\mathrm{CCH}$ will be available all the time to carry the messages exchanged between neighboring vehicles, as opposed to vehicles endowed with a single radio transceiver where a periodic switching between $\mathrm{CCH}$ and $\mathrm{SCH}$ is required [9]. The standard IEEE $802.11 \mathrm{p}$ developed specifically to enable Wireless Access in Vehicular Environments (WAVE) [9] is chosen as MAC protocol. This choice is justified by the high popularity of this standard protocol, particularly in the US and EU. Furthermore, communications in realistic scenarios are performed in the licensed frequency band of $5.9 \mathrm{GHz}$.

In order to implement our approach, we propose a new beacons format. In addition to the standard header of an IEEE802.11 MAC frame, we dedicate a part of the available 2312-bytes data field for the vehicles and the TLCs beacon payload. If needed, the payload will include adapted 
authentication and data security mechanisms since the IEEE 802.11 standard mechanisms are not used in IEEE 802.11p [9]. We depict in Figures 3(a) and 3(b) two wireless frames representing the beacons broadcast by the TLC and individual vehicles respectively.

The TLC beacon carries information about the sender TLC (its ID), the current traffic light state (i.e. green or red) and the remaining time in seconds before this state changes. It also holds information about unplanned incidents (e.g., vehicle collisions or accidents) or planned incidents (e.g., concerts or sporting events) ahead. This information will be relayed by the slave TLCs as well to vehicles in their road segments to self-adapt appropriately to any potential delays, e.g., make improved rerouting decisions. When the Smart Adaptation Engine recommends a new driving policy, this policy is advertised in the TLC beacon by indicating the policy code, to which driving direction it is applied and which lanes are concerned (in case of multiple lanes in one direction), as well as its validity time in seconds.

Regular beacons broadcast by vehicles are mainly used for neighborhood awareness purpose as well as to share individual desires and intentions. For instance, the entity type field is used to identify the sender vehicle special characteristics as some categories of vehicles may require particular actions/decisions to be taken such as ambulances and police cars. The remaining fields are used to store important information, including the Vehicle Identification Number (VIN), its current speed, its position in a given lane and its destination. The last field serves to share the vehicle desires and intentions by advertising up to eight desired actions. Each action is recognized by its code and corresponding value. An action value identifies the type of action to undertake (such as acceleration, deceleration, and changing lane), whereas its code could be either 0,1 , or 2 . An action code equals to 0 indicates that the vehicle has no interest to achieve the action or the action is already completed, the code 1 indicates the vehicle willingness to do the action (desire), and 2 means that the vehicle decided to take the action (intention). To protect the vehicle privacy and prevent tracking its location the VIN is not a constant value but instead a frequently changing pseudonym computed following a robust pseudonyms generation scheme such as [15].

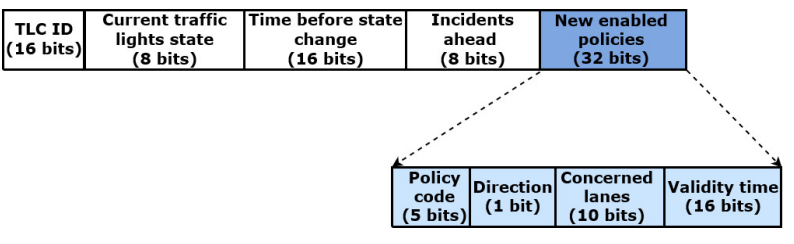

(a) TLC beacon format

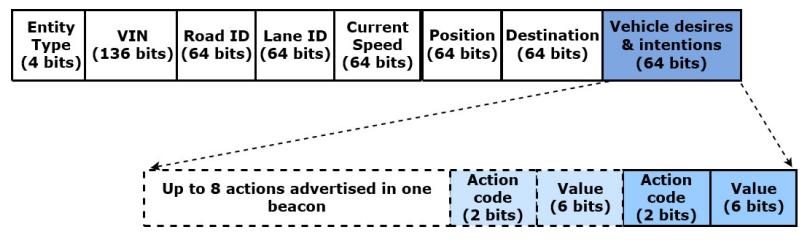

(b) Regular beacon format

Figure 3: TLC and regular beacon formats used in our solution
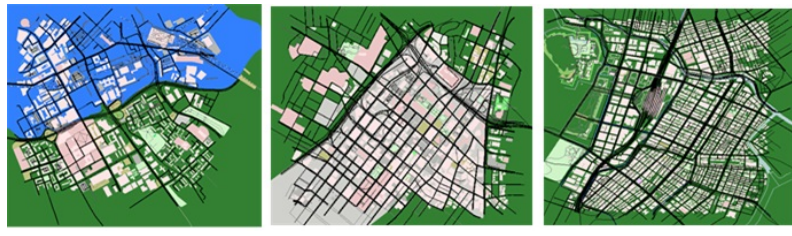

Figure 4: The three maps used in our simulation: from left to right, Manchester, Los Angeles and Tokyo

\section{Performance evaluation}

In this section, we present the performance evaluation results of our solution under three different sections of representative road layouts: Manchester, Los Angeles, and Tokyo (see Figure 4). The primary goal for this was to test how our solution's performance would be affected by the road layout.

A prototype of the proposed solution was implemented using the traffic simulator SUMO and TraCi client application. In our simulation, each road map is tested under varying vehicle densities: 400 vehicles (low density), 600 vehicles (medium density), and 800 vehicles (high density), and every vehicle is assigned a random route. In our simulation, we generated a mix of cars, buses, and trucks to mimic a realworld road network traffic. Before running the simulation to evaluate the efficiency of our solution, a baseline test was generated to measure the achieved average travel time in the three road maps and under the same three levels of traffic densities. This baseline test represents a road network controlled by regular TLCs that do not run our solution and do not exchange any traffic related information among each other, meaning that no adaptations like the ones recommended by the Smart Adaptation Engine, as explained in Section III-C, can be performed during the simulation when the baseline test is run. All simulations were run 20 times with different random seed values to get statistically meaningful results that are reproducible. In total, we used 20 different seed values, one value for each simulation run.

The obtained results of the achieved Average Travel Time (ATT) and average ATT improvement in our simulation are summarized in Table I and Figure 5, respectively. These results highlight that although the travel time reduction achieved by our solution, in Manchester map, was not significant in lowdensity scenario ( $1.7 \%$ only), it has increased to reach $4.5 \%$ under medium and high densities. Since the simulated section of Manchester map (the famous Oxford road) is a straightline road with limited number of traffic lights, the achieved improvement (i.e. reduction) in the ATT was limited to almost $5 \%$ in the best case. This result confirms our expectations as the impact of our solution is anticipated to be more significant when the number of junctions (traffic lights) is higher.

The histogram shown in Figure 5 reflects that the ATT measured in the simulated section of Los Angeles road network has a similar trend as the simulations with the Manchester map. However, results in this case show much higher improvement, especially in medium and high-density scenarios (up to 14\%). Here, the number of traffic lights is higher than in the Oxford road, which gives the master TLCs more opportunities to send the information regarding the remaining time for traffic 


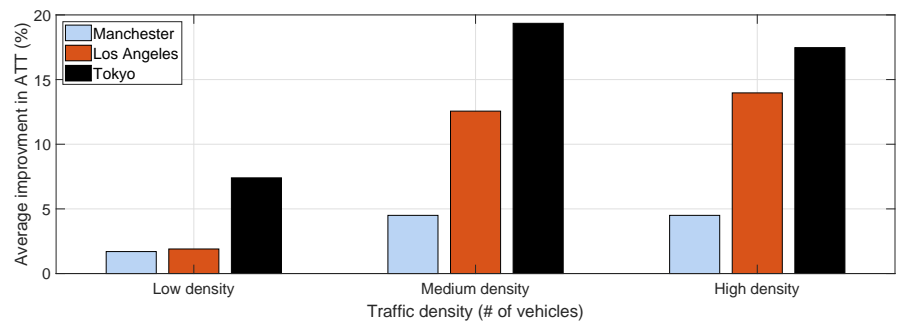

Figure 5: Average improvement in ATT achieved by our solution in the three road maps

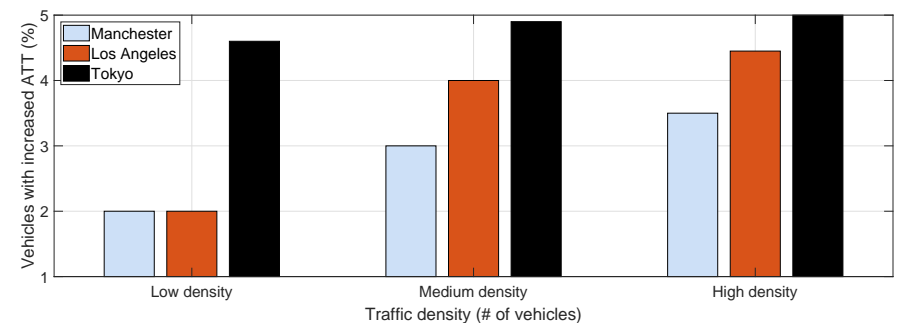

Figure 6: Impact of our solution on vehicles' individual efficiency in terms of their increased ATT

cycle, updated policies, etc. towards the vehicles that, in turn, adapt their speed accordingly and take some actions, whenever possible, to reduce their travel time to destination. Finally, in the same Figure, we observe that the ATT results for the simulated road section of Tokyo map show a significant reduction even in low-density scenario $(7.4 \%)$. This reduction in ATT further increases in medium and high densities to reach $19.35 \%$ and $17.47 \%$, respectively. We argue that this significant improvement is achieved because this road network is dense and has large number of traffic lights, thus the vehicles have more opportunities to benefit from our solution.

Figure 6 shows the percentage of vehicles that have seen an increase in their ATT when our solution is used. The plotted results indicate that the vehicles affected by such increase in ATT do not exceed 3.5\% in Manchester, $4.5 \%$ in Los Angeles, and $5 \%$ in Tokyo. These vehicles are usually the ones that were involved in a large number of collaborative actions to achieve a global benefit at the expense of their individual gain. Since our solution has achieved a positive improvement in ATT across all scenarios in the three road maps, this measured negative impact on the individual efficiency of some vehicles can be considered marginal given that at least $95 \%$ of other vehicles have seen an improvement in their ATT.

Please note that the above tests are carried out under the assumption that the penetration rate of autonomous vehicles is $100 \%$ and that the required smart infrastructure is already deployed. Therefore, the obtained results may vary if these assumptions are not in place. Any variation in the penetration rate and any missing component in the deployed infrastructure will certainly affect, at different degrees, the achieved results in terms of efficient collaboration between vehicles as well as their capabilities in communicating with the TLC and thus interpreting its transmitted updates of driving policies. Moreover, drivers' behaviours (if the penetration rate of autonomous vehicles is lower than 100\%) and their driving patterns may significantly affect the efficiency and feasibility of the proposed system. Accurately studying and quantifying such impact would require multi-disciplinary efforts, which is beyond the scope of this work.

\section{CONCLUSION}

In this paper, we studied the road traffic congestion issue and proposed a novel solution that aims to foster autonomous vehicles collaboration and ultimately reduce their travel time. Our solution is built upon an extended Belief-Desire-Intention (BDI) framework whereby vehicles can take collaborative actions for their individual and mutual benefits. To achieve this goal, vehicles are supported by an advanced road infrastructure that could intelligently reason on ongoing events and road settings to monitor road traffic and recommend actions to vehicles. Our solution leverages the use of the WAVE standard to optimize the usage of the available road infrastructure. It proposes a slight modification to the standard beacon format which is periodically broadcast by autonomous vehicles for neighborhood awareness purposes. It also proposes the usage of a new beacon designed for the TLC to communicate relevant information as well as temporary update in driving policies. The preliminary results of our solution prototype under three representative road maps have proven its efficiency. As a future work, we plan to extend our solution to accommodate the strict requirements of emergency vehicles while maintaining a tolerated impact on traffic conditions on road network and mitigate any potential risk of accidents.

\section{REFERENCES}

[1] D. Schrank et al., "2015 URBAN MOBILITY SCORECARD," Published jointly by The Texas A\&M Transportation Institute and INRIX, August 2015.

[2] S. Djahel et al., "A Communications-oriented Perspective on Traffic Management Systems for Smart Cities: 


\begin{tabular}{|c|c|c|c|c|c|c|}
\hline \multirow{2}{*}{ Traffic density } & \multicolumn{6}{|c|}{ Average Travel Time in seconds } \\
\cline { 2 - 7 } & \multicolumn{2}{|c|}{ Manchester } & \multicolumn{2}{c|}{ Los Angeles } & \multicolumn{2}{c|}{ Tokyo } \\
\cline { 2 - 7 } & Baseline & Our solution & Baseline & Our solution & Baseline & Our solution \\
\hline Low & 136.5 & 134.16 & 838 & 822.33 & 768.66 & 711.33 \\
\hline Medium & 149 & 142.2 & 1046.25 & 914.75 & 840.33 & 677.66 \\
\hline High & 151.2 & 144.4 & 1193.5 & 1026.75 & 933 & 770 \\
\hline
\end{tabular}

Table I: The achieved ATT (in seconds) in the three road maps: Baseline vs. our solution

Challenges and Innovative Approaches," IEEE Communications Surveys and Tutorials, Vol. 17, No. 1, 2015.

[3] S. Wang et al., "Next Road Rerouting: A Multi-Agent System for Mitigating Unexpected Urban Traffic Congestion," IEEE Transactions on Intelligent Transportation Systems, 2016.

[4] S. Wang et al., "An Adaptive and VANETs-based Next Road Re-routing System for Unexpected Urban Traffic Congestion Avoidance," IEEE VNC 2015, Dec. 16-18, 2015, Kyoto, Japan.

[5] C. Wu et al.,"Flow: Architecture and Benchmarking for Reinforcement Learning in Traffic Control," CoRR, abs/1710.05465, 2017, http://arxiv.org/abs/1710.05465

[6] Richard W. Willson, "Estimating the Travel and Parking Demand Effects of Employer-Paid Parking," Regional Science and Urban Economics, Vol. 22, 133-145, 1992.

[7] E. Uhlemann, "Connected-Vehicles Applications Are Emerging [Connected Vehicles]," in IEEE Vehicular Technology Magazine, vol. 11, no. 1, pp. 25-96, Mar. 2016.

[8] S. Haykin, "Cognitive radio: brain-empowered wireless communications," in IEEE Journal on Selected Areas in Communications, vol. 23, no. 2, pp. 201-220, Feb. 2005.

[9] IEEE Standard for Wireless Access in Vehicular Environments (WAVE)- Multi-Channel Operation, IEEE Vehicular Technology Society. IEEE Std 1609.4-2016 (Revision of IEEE Std 1609.4-2010)

[10] H. Hu et al., "REPLACE: A Reliable Trust-based Platoon Service Recommendation Scheme in VANET," IEEE Transactions on Vehicular Technology, vol. 66, no. 2, pp. 1786- 1797, Feb. 2017.

[11] A.M. Thomson et al., "Conceptualizing and measuring collaboration," Journal of Public Administration Research and Theory, 19(1): 23-56, 2009.

[12] Bedwell, W.L., et al., "Collaboration at work: An integrative multilevel conceptualization," Human Resource Management Review (2012), doi: 10.1016/j.hrmr.2011.11.007

[13] O. Adam et al., "A Collaboration Framework for Crossenterprise Business Process Management," First International Conference on Interoperability of Enterprise Software and Applications- INTEROP-ESA, pp: 499510, 2005.

[14] S. Djahel et al., "Toward V2I communication technologybased solution for reducing road traffic congestion in smart cities," In Networks, Computers and Communications (ISNCC), 2015 International Symposium on , vol., no., pp.1-6. 2015.
[15] M. Gerlach and F. Guttler, "Privacy in VANETs using Changing Pseudonyms - Ideal and Real," 2007 IEEE 65th Vehicular Technology Conference - VTC2007-Spring, Dublin, 2007, pp. 2521-2525.

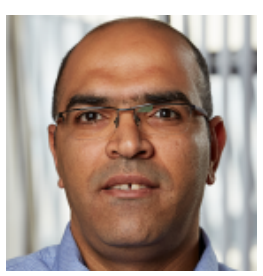

Soufiene Djahel (SM-IEEE) received the M.Sc. degree in computer science from the University of Bejaia, Algeria, in 2007, and the Ph.D. degree in computer science from Lille 1 University of Science and Technology, France, in 2010. He has been a Senior Lecturer with the department of Computing and Mathematics, Manchester Metropolitan University, U.K., since 2015. His main research interests include Security and QoS issues in wireless networks, Intelligent Transportation Systems (ITS), and e-health. Since 2008, he has published more than 50 peer reviewed conference and journal papers in the reputable IEEE Core/Flagship conferences and journals in wireless networks and ITS research domain.

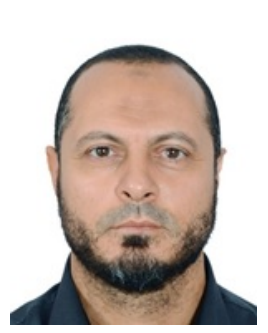

Nafaa Jabeur is Associate Professor and Director of Research at the German University of Technology in Oman (GUtech). He received his Master degree and his $\mathrm{PhD}$ degree in Computer Science from Laval University (Quebec, Canada) in 2001 and 2006 respectively. He has over than 12 years of experience in the industrial and academic sectors. Nafaa has participated in several R\&D projects, authored 2 books, and authored more than 80 research papers in prestigious conferences and high ranked journals. $\mathrm{He}$ is active researcher in a variety of fields, including Drones, Internet of Things, Smart Cities, Artificial Intelligence, Mobility and Transportation, and Spatial Data Warehouses.

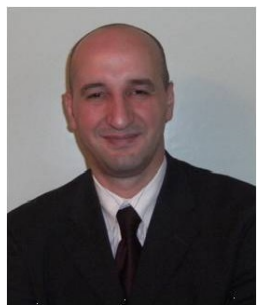

Farid Nait-Abdesselam received his B.Sc. degree from Bab Ezzouar University, Algeria, in 1993 his M.Sc. degree from Paris Descartes University, France, in 1994, and his Ph.D. degree from Versailles University, France, in 2000. He is a Professor at University of Missouri Kansas City. His research interests lie in the field of computer and communication networks with emphasis on algorithms, architectures and protocols for security and optimization in wireless and wired networks. Farid Nait-Abdesselam is a Senior Member of IEEE.

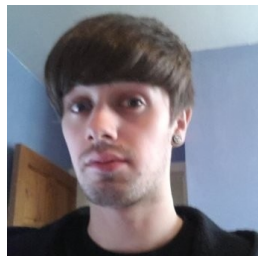

Thomas Wolstencroft received a Bachelor in Computer Science Degree from Manchester Metropolitan University in 2016. Currently, he is running a family business. 